\title{
Newborns with Delayed Hearing Screening Prior to Hospital Discharge: High Risk Populations of Hearing Loss
}

\author{
Mahmoud A. Gaddoury*1, Tri Tran², 3, Mary Jo Smith², Jeanette Webb², Terri Mohren² and \\ Melinda Peat ${ }^{2}$
}

${ }^{1}$ Tulane Univrsity, New Orleans, LA, USA; 'Louisiana DHH OPH CSHS Early Hearing Detection and Intervention Program, New Orleans, LA, USA; ${ }^{3}$ LSUHSC School of Medicine, Department of Pediatrics, New Orleans, LA, USA

\section{Objective}

The objective of the study was to compare the average length of time for hearing screening to be conducted prior to hospital discharge with newborns' characteristics among birth cohort of 2011 in Louisiana.

\section{Introduction}

Hearing loss is the most common birth defect, occurring at a rate of 1-3 of every 1000 births. The goal of the Louisiana Early Hearing Detection and Intervention Program (LA EHDI) is to ensure that all newborns are screened for hearing loss so that those with a diagnosis of permanent hearing loss can achieve their maximum potential.

LA EHDI promotes the national EHDI goals of screening for hearing loss by 1 month of age, preferably before hospital discharge; completing diagnostic evaluations for all infants who screen positive by 3 months of age; and for all infants identified with hearing loss, receiving appropriate early intervention services by 6 months of age. The findings of this study are expected to help LA EHDI define characteristics of newborns who receive late initial hearing screening tests to be performed in the hospital. The program may consider if those characteristics are potentially related to hearing loss so that appropriate plans to prevent risk factors of hearing loss are developed appropriately

\section{Methods}

LA EHDI initial hearing screening data were linked with Louisiana birth certificates of newborns born in 2011. All newborns with no screening tests were excluded from the study. The length of time for receiving hearing screening was defined as the number of days between the date of birth and the date of screening before hospital discharge. All variables defining newborns' characteristics were derived from birth certificates and included birth weight, gestational age, admission to NICU, Apgar score at 5th minute after birth, method of delivery, and plurality. Linear regression models were used to compare the length of hearing screening between subgroups in each characteristic. In adjusted models, all variables were included and controlled for. Data analyses and linkage were conducted with SAS 9.3 and Linkpro 3.0. Alpha was set at .05 for statistical significance.

\section{Results}

57,942 newborns who met inclusion criteria were included in the study. The length was highest among newborns with very low birth weight (VLBW, < 1,500 grams) (mean in days [M]: 41.7, CI95\% [CI]: 40.1-43.4], followed by newborns with very preterm (VPT, < 32 weeks of gestational age) (M: 38.1, CI: 36.5-39.6), those who admitted to NICU (M: 12.9, CI: 12.4-13.3), those with low Apgar score $(<7)$ at 5 th minute after delivery (M: 12.0, CI: 10.6-13.5). In adjusted linear regression model, the length was 22.2 days (CI: 21.922.6) higher among newborns with VLBW than among normal birth weight newborns; and 16.8 days (CI: 16.5-17.3) higher among those with PTB than among term birth newborns. It was found that the length was 4.7 days (CI: 4.6-4.9) higher among newborns who admitted to NICU than among those who did not; and 1.8 days (CI: 1.52.0) higher among newborns who had low Apgar score than among counterparts who had normal score. The length was not found much different among newborns who had different methods of delivery, as well as among singleton or twin/triplet newborns.

\section{Conclusions}

Newborns who had very low birth weight, were very preterm, admitted to NICU, or had low Apgar score received late screening in hospital prior to discharge. These characteristics have been defined as risk factors of hearing loss. Effective plans and strategies to improve adverse birth outcomes and delivery status of newborns are also to identically improve late receipt of initial screening as well as to reduce risk factors of hearing loss.

\section{Keywords}

EHDI; Hearing Loss; Delayed Hearing Screening

\author{
*Mahmoud A. Gaddoury \\ E-mail: mgaddour@tulane.edu
}

